\title{
Crossed Andreev reflection in InSb flake Josephson junctions
}

\author{
Folkert K. de Vries, ${ }^{1, *}$ Martijn L. Sol, ${ }^{1}$ Sasa Gazibegovic, ${ }^{2}$ Roy L. M. op het Veld, ${ }^{2}$ Stijn C. Balk, ${ }^{1}$ Diana Car, ${ }^{2}$ \\ Erik P. A. M. Bakkers, ${ }^{2}$ Leo P. Kouwenhoven, ${ }^{1,3}$ and Jie Shen ${ }^{1, \dagger}$ \\ ${ }^{1}$ QuTech and Kavli Institute of Nanoscience, Delft University of Technology, 2600 GA Delft, The Netherlands \\ ${ }^{2}$ Department of Applied Physics, Eindhoven University of Technology, $5600 \mathrm{MB}$ Eindhoven, The Netherlands \\ ${ }^{3}$ Microsoft Quantum Lab Delft, 2628 CJ, Delft, The Netherlands
}

(Received 13 June 2019; revised manuscript received 12 September 2019; published 4 December 2019)

\begin{abstract}
We study superconducting quantum interference in InSb flake Josephson junctions. An even-odd effect in the amplitude and periodicity of the superconducting quantum interference pattern is found. Interestingly, the occurrence of this pattern coincides with enhanced conduction at both edges of the flake, as is deduced from measuring a superconducting quantum interference device (SQUID) pattern at reduced gate voltages. We identify the specific crystal facet of the edge with enhanced conduction, and confirm this by measuring multiple devices. Furthermore, we argue the even-odd effect is due to crossed Andreev reflection, a process where a Cooper pair splits up over the two edges and recombines at the opposite contact. An entirely $h / e$ periodic SQUID pattern, as well as the observation of both even-odd and odd-even effects, corroborates this conclusion. Crossed Andreev reflection could be harnessed for creating a topological state of matter or performing experiments on the nonlocal spin entanglement of spatially separated Cooper pairs.
\end{abstract}

DOI: 10.1103/PhysRevResearch.1.032031

Induced superconductivity in semiconductors with strong spin-orbit interaction (SOI) has attracted much interest for its potential applications in topological quantum computation [1]. A semiconducting Josephson junction (JJ) offers a platform to study the induced superconductivity by means of superconducting quantum interference (SQI) [2]. Recently, induced superconductivity in edge channels in the quantum Hall regime [3] and in a predicted two-dimensional topological insulator [4,5], interesting for topological zero modes such as parafermions or Majoranas, are investigated using SQI. Additionally, an oscillation with both $h / e$ and $h / 2 e$ periodic components, before connected to topological edge states [5], is observed in a trivial InAs quantum well and attributed to crossed Andreev reflection (CAR) in the JJ [6,7].

Crossed Andreev reflection is a process where the quasiparticles that form a Cooper pair are spatially separated but still entangled. The entanglement of these quasiparticles holds promise in harnessing electrons in a solid-state environment to, for example, test the Einstein-Podolsky-Rosen paradox [8] —of fundamental importance to both quantum communication and computation. Additionally, coupling two onedimensional (1D) structures (i.e., nanowires or edge states)

\footnotetext{
*Present address: Solid State Physics Laboratory, ETH Zürich, CH8093 Zürich, Switzerland.

†resent address: Beijing National Laboratory for Condensed Matter Physics, Institute of Physics, Chinese Academy of Sciences, Beijing 100190, China; shenjiexx@126.com

Published by the American Physical Society under the terms of the Creative Commons Attribution 4.0 International license. Further distribution of this work must maintain attribution to the author(s) and the published article's title, journal citation, and DOI.
}

via CAR is interesting for engineering a topological state of matter hosting parafermions [9] or Majoranas [10]. To observe pronounced CAR in a device, normal or direct Andreev reflection needs to be suppressed. In this regard, quantum dots [11,12] or Luttinger liquids [13] can be utilized. Twodimensional (2D) systems, such as a 2D electron gas [14] connected to a superconductor, offer a scalable and flexible platform for more complex device geometries. Therefore, exploiting coupled 1D edge channels in a 2D material for Cooper pair splitting combines the large CAR amplitude and flexibility in device design $[6,15]$.

Here, we obtain measurements of CAR in a JJ made of an InSb flake, a 2D nanostructure. We observe both even-odd and odd-even Fraunhofer patterns, and an entirely $h / e$ periodic superconducting quantum interference device (SQUID) pattern in JJs with enhanced conduction at both edges. We argue that these $h / e$ effects are caused by a flux-independent supercurrent due to CAR, where the quasiparticles are spatially separated over the two edges.

$\mathrm{InSb}$ is known for its large $g$-factor [16] and strong SOI [17], and earlier works referred to the flakes as nanosails [16] or nanosheets $[18,19]$. The InSb flakes (Fig. 1) are grown with the vapor-liquid-solid technique [20]. The crystal facets of the flake can be deduced from the facets of the nanowires it originates from. The nanowire has (110) facets, and the facets of the flake that are parallel to one of the two nanowires therefore have these (110) facets as well, as sketched in Fig. 1(b). This is the case for both edges in $\mathrm{JJ} 1$ and only one edge of $\mathrm{JJ} 2$, for example [Fig. 2(a)]. The (110) facet is known for having electron accumulation at its surface $[21,22]$, because the lack of $\mathrm{Sb}$ atoms results in band bending [23]. Considering the geometry, we expect strong band bending at the edges of the flake with (110) facets, as sketched in Figs. 2(a) and 2(b). To fabricate devices, we use a micromanipulator to transfer the flakes to a 
(a)

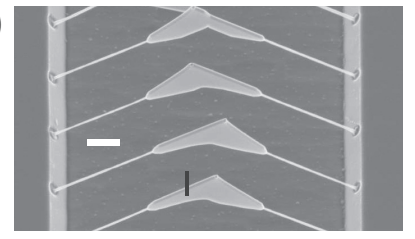

(b)

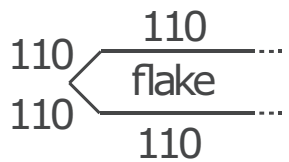

FIG. 1. (a) A scanning electron microscope (SEM) image of the InSb flakes, grown from two nanowires on an InP substrate [20]. The scale bar represents $500 \mathrm{~nm}$. (b) Sketch of the cross section of the flake, at the location highlighted by the solid black line in (c). All facets, top, bottom, and edge, have (110) crystal facets. Note that these are the edge facets stemming from the nanowire.

$\mathrm{Si} / \mathrm{SiO}_{x}$ substrate that serves as a global bottom gate. Then, two NbTiN contacts are deposited after treating the surface with a sulfur solution to remove the native oxides $[24,25]$. The geometrical parameters, such as contact separation $L$, and width $W$, of all JJs are presented in the Supplemental Material [26]. The JJs are measured in a quasi-four-terminal current bias setup at a temperature of $300 \mathrm{mK}$, unless stated otherwise. Characterization of the superconductivity provides us an estimate of the superconducting gap $\Delta$ of $1.4 \mathrm{meV}$, consistent with values found earlier for NbTiN [25], and the induced superconducting coherence length $\xi_{s}$ of $1.2 \mu \mathrm{m}$ at $V_{\mathrm{BG}}=15 \mathrm{~V}$ (see Supplemental Material for details [26]).

Superconducting quantum interference measurements are performed by measuring the switching current of the JJs while varying the flux through them with an out-of-plane magnetic field. Interestingly, the SQI pattern of JJ1 in Fig. 2(c) does not show the regular Fraunhofer pattern [2]. The pattern instead displays an even-odd effect, which means the amplitude of the side lobes is not monotonically decaying but alternating. The first side lobe has a smaller amplitude than the second, and the amplitude of the third side lobe is zero. Such a even-odd behavior has been predicted before by Barzykin et al., for junctions with a large length over width ratio [27]. This served as an explanation for a doubling of the period in such a junction [28]. In this model the supercurrent is calculated over all possible quasiparticle trajectories for either a ballistic or diffusive junction. Here, we start off with the ballistic model, since we estimate the mean free path $l_{\mathrm{MFP}}$ to be around $250 \mathrm{~nm}$, similar to $L$ [Fig. 4(b)]. The expressions for the resulting SQI patterns for both cases are provided in the Supplemental Material for convenience [26]. Plugging in our device parameters, we find, however, that the model proposed by Barzykin et al. only is not sufficient to reproduce our even-odd Fraunhofer pattern in Fig. 2(c). We have to add an additional offset, $f=0.09$, to the model,

$$
I_{c}(\Phi)=I_{c 0}|\mathcal{I}(\Phi)+f|,
$$

where $I_{c 0}$ is the critical current at zero magnetic field, and $\Phi$ the flux through the junction. The offset increases (decreases) the amplitude of the lobes with the same (opposite) sign. We thus describe the even-odd effect with a positive,

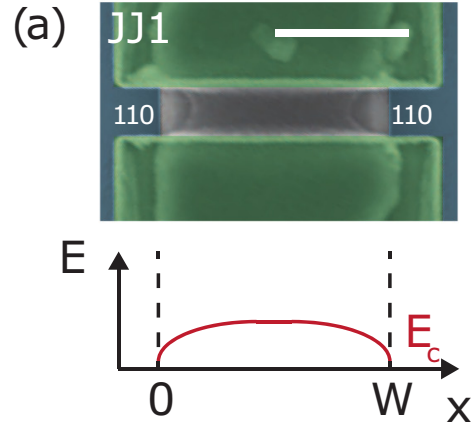

(b)

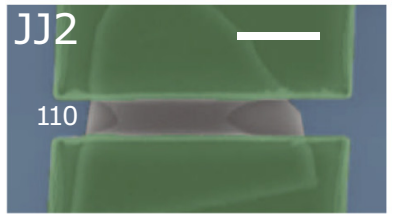

E

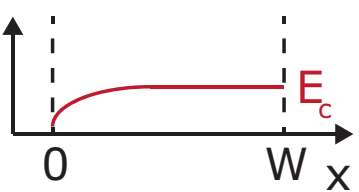

(c)

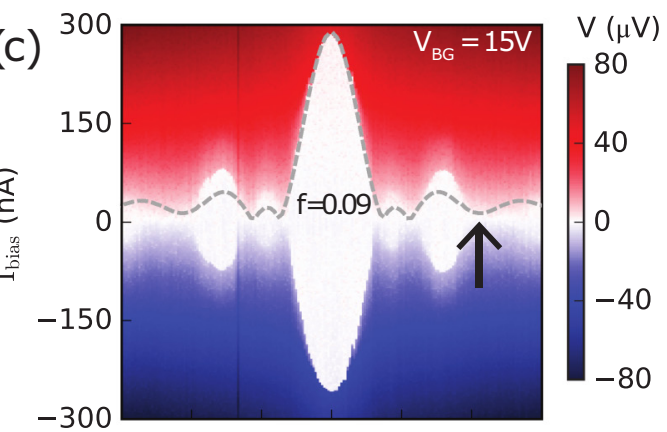

(d)
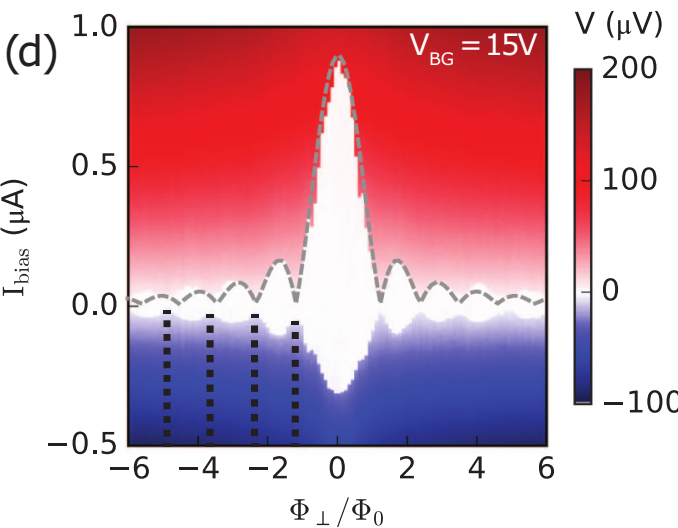

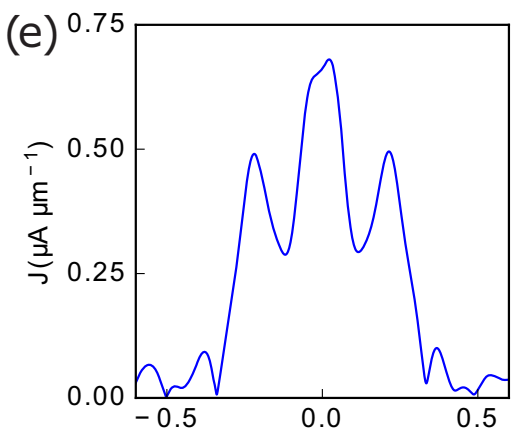

(f)

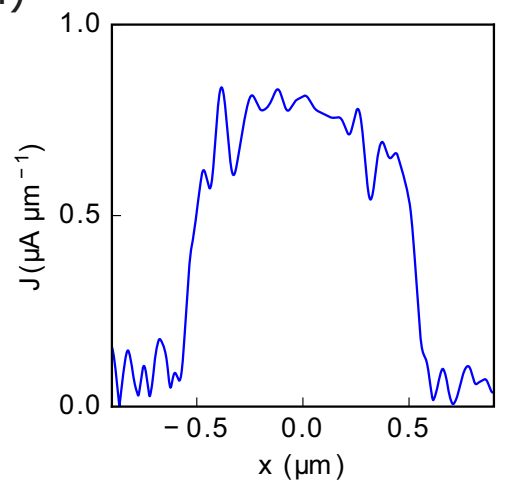

FIG. 2. (a), (b) The upper panel shows a false colored scanning electron microscope image of JJ1 and JJ2, respectively. The flake (gray) is deposited on a $\mathrm{Si}_{2} / \mathrm{SiO}_{x}$ substrate (blue) and contacted by NbTiN (green). The scale bar represents $500 \mathrm{~nm}$. In the bottom panel, the energy of the bottom of the conduction band $E_{c}$ (red) is sketched along the width $W$ of the junction. (c), (d) Voltage $V$ measured over JJ1 and JJ2 as a function of current bias $I_{\text {bias }}$ and normalized flux $\Phi / \Phi_{0}$ with $\Phi_{0}=h / 2 e$, through the $\mathrm{JJ}$ area, at a bottom gate voltage $V_{\mathrm{BG}}$ of $15 \mathrm{~V}$. The dashed gray line is a calculated SQI pattern following Ref. [27] with an offset $f=0.09$ for (c) and no offset for (d) [26]. For (c), the arrow highlights the missing third side lobe and in (d), the periodicity of the SQI pattern is indicated by the dashed black lines. (e), (f) Current density distribution $J$ extracted from the SQI pattern of (c) and (d), using the Dynes-Fulton approach [29]. 


\section{(a)}
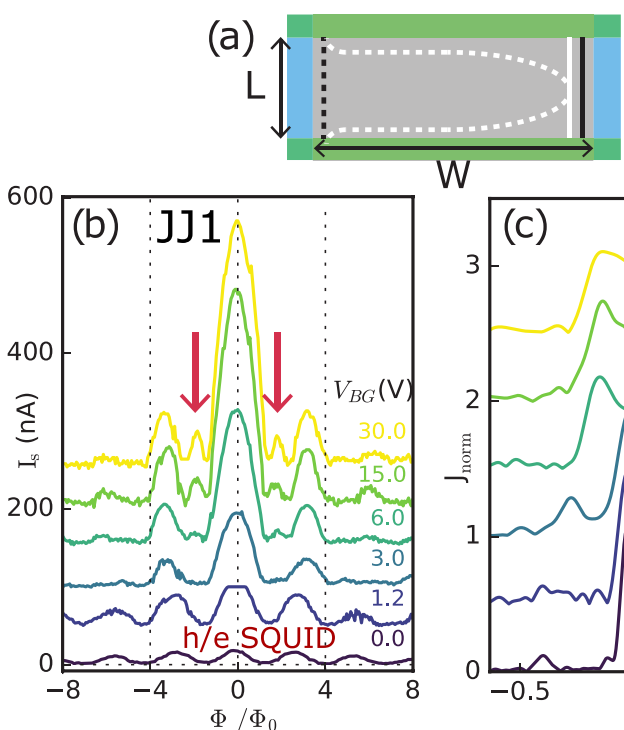

W
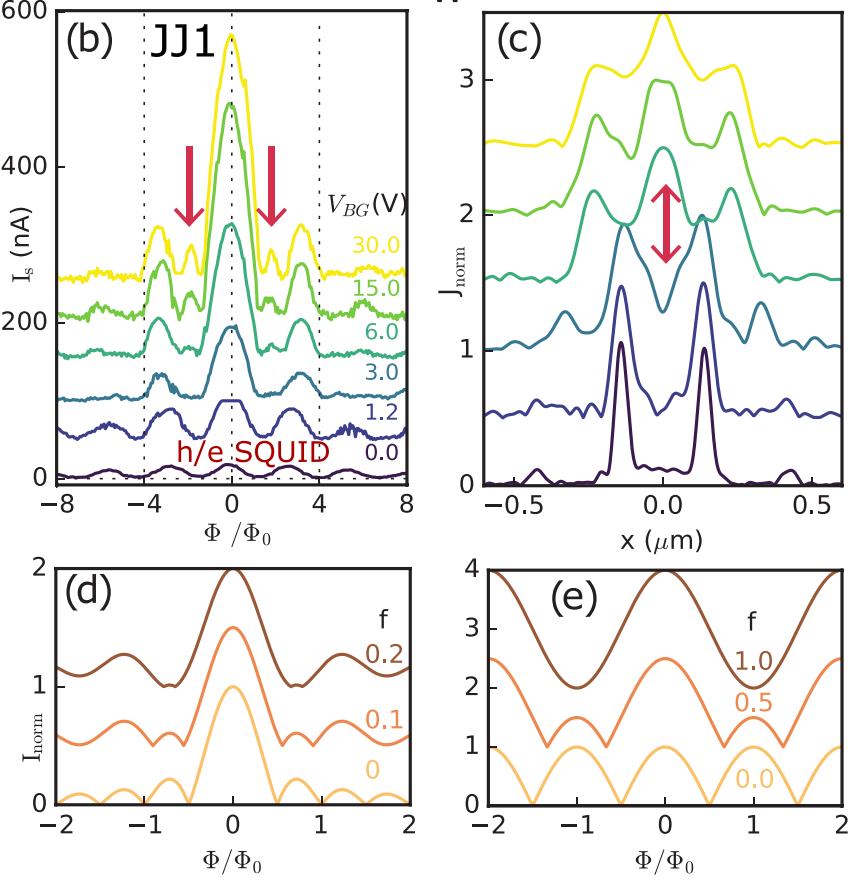

FIG. 3. (a) A sketch of crossed and direct Andreev reflection. The dashed (solid) line schematically represents a crossed (normal) Andreev reflection. The electron (white) encircles the junction, and picks up a phase due to flux $\Phi$ while the hole (black) flows along the opposite edge. (b) Switching current $I_{\mathrm{s}}$ as a function of normalized perpendicular magnetic flux $\Phi / \Phi_{0}$ for JJ1 at the indicated gate voltages $V_{\mathrm{BG}}$. The red arrows highlight the first lobe, whose amplitude is diminished as $V_{\mathrm{BG}}$ decreases. (c) Normalized current density distributions $J_{\text {norm }}$ extracted from the SQI patterns from (b). The disappearance of the central peak is highlighted by the red arrow. (d), (e) Calculated SQI patterns with a positive offset $f$ for a standard Fraunhofer and SQUID pattern, respectively [26].

magnetic-field-independent supercurrent offset $f$ added to the expected interference pattern $\mathcal{I}(\Phi)$. Examples of Fraunhofer and SQUID patterns with different positive offsets are presented in Figs. 3(d) and 3(e). A SQUID pattern with such an offset has been reported before [5,7], however, the even-odd Fraunhofer pattern still needs to be experimentally studied.

For JJ2, the SQI pattern can also not be described by the standard Fraunhofer pattern [2], because our JJs does not satisfy the limit of $W \gg L(W=1280 \mathrm{~nm}$ and $L=240 \mathrm{~nm})$. Note that all SQI patterns have been compensated already for flux focusing due to the Meissner effect [26]. In contrast to $\mathrm{JJ} 1$, the SQI pattern of JJ2 is well resembled by the calculated SQI pattern [Fig. 2(d)], showing that the larger periodicity of $1.5 \Phi_{0}$ is due to the rectangular geometry of the JJ [27,28].

In the calculations we have implicitly taken into account that the supercurrent is homogeneously distributed throughout the JJ. To check this, we reconstruct the current density distribution with the method described by Dynes and Fulton [29], which we are allowed to use, since the JJs are in the short junction limit $\xi_{s}>L$ [30]. The current density distribution for JJ2, plotted in Fig. 2(f), is homogeneously distributed, whereas for JJ1 [Fig. 2(e)] it reveals a large peak at the center of the JJ. Apart from having a supercurrent through the center of the JJ, the peak could also be due to a magnetic-fieldindependent supercurrent. Because the Dynes-Fulton method is based on a Fourier transform, constant (or zero-frequency) components end up at $x=0$, the center of the distribution. Such a supercurrent offset cannot be due to a partial short in the JJ, since we confirmed the supercurrent can be pinched off by the global bottom gate [26]. Because of its insensitivity to the magnetic field, the offset cannot stem from mechanisms that occur at a certain magnetic field either [31,32]. An effect that however could cause a magnetic-field-independent supercurrent is CAR [11]. CAR describes an Andreev pair of which one quasiparticle encircles the junction area [Fig. 3(a)] and therefore acquires a phase proportional to the flux through the junction area. That extra phase can either directly [33], or by interference of two different Andreev pairs, result in a flux-independent supercurrent $[6,34,35]$.

To find out whether a central current path or a magneticfield-independent supercurrent due to CAR causes the evenodd effect, we continue by studying the gate dependence of the SQI patterns. The even-odd SQI pattern from JJ1 changes drastically as a function of gate voltage [Fig. 3(b)]. The amplitude of the first side lobe decreases as $V_{\mathrm{BG}}$ is reduced (highlighted by the red arrows), and becomes zero at $V_{\mathrm{BG}}=$ $3 \mathrm{~V}$. Then, for the bottom two traces of Fig. 3(b), the SQI pattern takes a cosinusoidal shape, known as a SQUID pattern [2]. When the amplitude of the second lobe drops below the offset of the SQI pattern, the periodicity of the SQI pattern changes [see also the curve for $f=1$ in Fig. 3(e)]. It doubles from $1.3 \Phi_{0}$ at $V_{\mathrm{BG}}=15 \mathrm{~V}$ to $2.7 \Phi_{0}$ at $V_{\mathrm{BG}}=3 \mathrm{~V}$, and the SQI pattern becomes entirely $h / e$ periodic for $V_{\mathrm{BG}} \leqslant 1.2 \mathrm{~V}$. This is different from the observed $h / e$ SQUID in Refs. [5,7], where the amplitude was larger than the offset and therefore an $h / 2 e$ oscillation is observed simultaneously. Our observation confirms that the $h / e$ periodicity is not a unique signature of a topological JJ [7].

The changes in the SQI pattern are reflected in the extracted current density distributions in Fig. 3(c). Note that $J$ is spanning half of the width for $V_{\mathrm{BG}} \leqslant 3 \mathrm{~V}$ compared to $V_{\mathrm{BG}} \geqslant 6 \mathrm{~V}$, because we used the same area and flux periodicity for the calculation of all traces. The center peak in the current density disappears at $V_{\mathrm{BG}}=3 \mathrm{~V}$. Such a local effect in $J$ is not likely to be caused by changing the global gate. Additionally, the offset in the SQI patterns persists, even though there is no longer any center peak. Therefore, we disregard a current path at the center of the $\mathrm{JJ}$ as an explanation for the even-odd effect.

Interestingly, between $V_{\mathrm{BG}}=3$ and $0 \mathrm{~V}$, the SQUID pattern translates to a current density distribution with edge conduction only [bottom trace of Fig. 3(c)], in agreement with JJ1 having electron accumulation at both edges [Fig. 2(a)]. This enhanced conduction at both edges of the JJ, in combination with the $h / e$ periodicity, is consistent with the occurrence of CAR $[6,7]$. To substantiate this, we consider this mechanism in detail, and study additional JJs.

The CAR trajectories encircling the JJ area consist of the edges of the flake, and two paths along the contacts, as sketched in Fig. 3(a). The latter arises from doping from the 

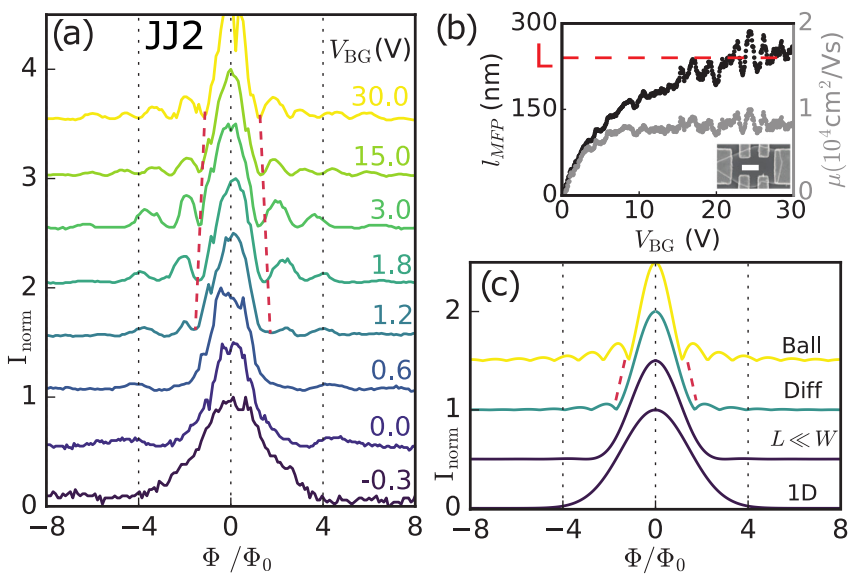

FIG. 4. (a) Normalized switching current $I_{\text {norm }}$ as a function of normalized flux $\Phi / \Phi_{0}$ for the indicated bottom gate voltages $V_{\mathrm{BG}}$. The dashed red lines highlight an increase in periodicity as $V_{\mathrm{BG}}$ is reduced. (b) Mean free path $l_{\text {MFP }}$ and mobility $\mu$ as a function of $V_{\mathrm{BG}}$, extracted from Hall measurements [26]. A SEM image of the Hall bar device is presented in the inset, with a scale bar representing $500 \mathrm{~nm}$. The length $L$ of $\mathrm{JJ} 2$ is indicated by the dashed red line for comparison. (c) The SQI pattern for a ballistic (Ball), diffusive (Diff), and quasi-1D $(L \gg W) \mathrm{JJ}$ are obtained from Ref. [27], and for a 1D junction from Ref. [37], details of which can be found in the Supplemental Material [26].

contact in combination with a finite barrier to the contact [7]. The estimated induced superconducting coherence length of $1.2 \mu \mathrm{m}$ is close to the typical junction circumference of $2 \mu \mathrm{m}$ [26]. Due to the difference between the circumference and $L$, the CAR is expected to be suppressed with temperature before direct Andreev reflection (and supercurrent) diminishes. A temperature dependence is however ambiguous, since the side lobes (and with that the even-odd effect) disappear before the switching current is suppressed [26]. The magnitude of the offset reaches a value of 1 in Fig. 3(b), which is in range of what one can expect for a combination of a small coupling to the contact, while maintaining the Fermi velocity in the edges [6,26]. Additionally, the electron-electron interaction in the $1 \mathrm{D}$ edges could also reduce the direct Andreev reflection and lead to a large $f$ [36]. The large offset $f$ and $h / e$ periodicity mean that the CAR amplitude exceeds the direct Andreev reflection, an interesting topic and regime for future experiments.

To shine light on the correlation between the even-odd effect and having enhanced conduction at both edges, we study the gate dependence of $\mathrm{JJ} 2$ as well. The periodicity of the SQI patterns in Fig. 4(a) grows slightly from $1.3 \Phi_{0}$ to $1.5 \Phi_{0}$ as $V_{\mathrm{BG}}$ is lowered from $30 \mathrm{~V}$ to $1.2 \mathrm{~V}$. Meanwhile the mobility $\mu$ and $l_{\mathrm{MFP}}$ decrease [Fig. 4(b)], and for $V_{\mathrm{BG}}<3 \mathrm{~V}$ the length $L$ of the $\mathrm{JJ}$ is larger than $l_{\mathrm{MFP}}$ and the JJ changes from ballistic to diffusive. In Fig. 4(c), calculated SQI traces for ballistic and diffusive transport are plotted [27], consistent with the transition observed in our data. Furthermore, the strong increase in periodicity and suppression of the side lobe amplitudes for gate voltages below $V_{\mathrm{BG}}=1.2 \mathrm{~V}$ highlight a transition from a $2 \mathrm{D}$ to a $1 \mathrm{D}$ diffusive regime. The measured $\mathrm{SQI}$ pattern at $V_{\mathrm{BG}}=-0.3 \mathrm{~V}$ is well reproduced by the theoretical curves for a (quasi-)1D JJ [Fig. 4(c)], as described
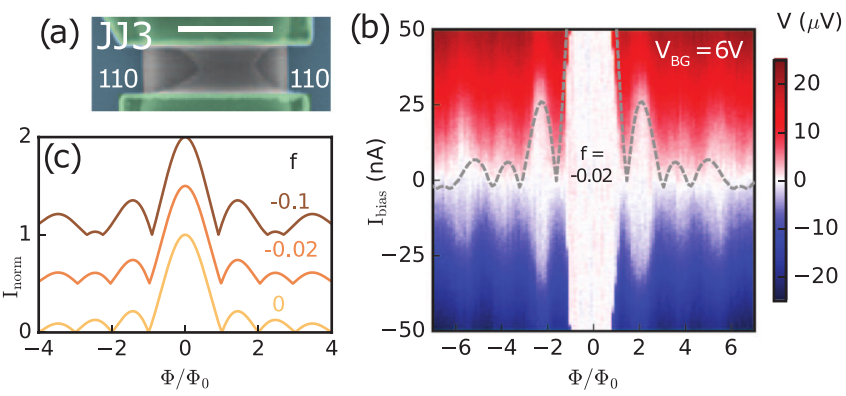

FIG. 5. (a) False colored SEM image of JJ3. The flake (gray) is deposited on a $\mathrm{Si} / \mathrm{SiO}_{x}$ substrate (blue) and contacted by NbTiN (green). The scale bar represents $500 \mathrm{~nm}$. (b) SQI pattern of JJ3 at $V_{\mathrm{BG}}=15 \mathrm{~V}$. The dashed gray line is a simulation following Ref. [27] with offset $f=-0.02$ [26]. (c) Calculated Fraunhofer patterns with a variable (negative) offset $f$, as indicated.

[27,37] and observed before [37,38]. Entering the 1D regime is in line with having a single edge with a (110) facet and enhanced edge conduction in JJ2 [Fig. 2(b)]. Continuing the argument, not finding an even-odd effect in $\mathrm{JJ} 2$ strengthens the connection between the even-odd SQI pattern and having enhanced conduction at both edges.

Two other devices (JJ3 and JJ4) also reveal an even-odd SQI pattern, and show enhanced conduction in both their edges with a (110) facet [26]. Furthermore, JJ5-JJ7, having a single (110) edge, do not show an even-odd SQI pattern [26]. Interestingly, the SQI pattern from JJ3 [Fig. 5(b)] shows a negative offset, or odd-even effect. The third lobe has a similar amplitude to the second, thus is not smaller as expected for a standard Fraunhofer pattern. By adding a negative offset to the calculated SQI pattern from Ref. [27] [dashed gray line in Fig. 5(b)], we indeed find good agreement with the data. For comparison we plotted the standard Fraunhofer patterns with negative offsets in Fig. 5(c). Having either a positive or negative offset to the switching current due to CAR depends on microscopic details regarding the spin mixing in the JJ [6]. To be more precise, spin mixing with predominantly spin-conserving or spin-flip processes refers to a positive or negative offset, respectively. In our InSb flakes, the spin mixing is probably caused by a strong spin-orbit interaction in the InSb [17]. The observation of a negative offset is, to our knowledge, unique to CAR [6], and therefore strongly supports that CAR is causing the observed $h / e$ periodic SQI patterns.

In conclusion, we observe $h / e$ effects due to crossed Andreev reflection in the SQI patterns of InSb flake Josephson junctions with enhanced conduction at both edges. We identified the (110) crystal facet to have enhanced edge conduction, and can thus in the future choose to either study or circumvent them. The observed $h / e$ SQUID pattern reveals that the CAR amplitude can exceed the direct Andreev reflection in a 2D semiconducting Josephson junction. The InSb flakes therefore provide a promising platform to use CAR for creating topological zero modes $[9,10]$ or for applications in Cooper pair splitting $[8,15]$.

The supporting data for this article are openly available from the 4TU.Centre for Research Data archive [39]. 
This work has been supported by funding from the Netherlands Organization for Scientific Research (NWO) and Microsoft. S.C.B. developed the substrate preparation, S.G. grew the flakes, D.C. and E.P.A.M.B. contributed to the growth, and R.L.M.o.h.V. developed the flake deposition procedure. J.S., F.K.d.V., and M.S. fabricated the devices, performed the measurements, and analyzed the data. F.K.d.V. and J.S. wrote the manuscript with comments from all authors. J.S. and L.P.K. supervised the project.
[1] J. Alicea, Rep. Prog. Phys. 75, 076501 (2012).

[2] M. Tinkham, Introduction to Superconductivity, Dover Books on Physics Series (Dover, New York, 1996).

[3] F. Amet, C. T. Ke, I. V. Borzenets, J. Wang, K. Watanabe, T. Taniguchi, R. S. Deacon, M. Yamamoto, Y. Bomze, S. Tarucha, and G. Finkelstein, Science 352, 966 (2016).

[4] S. Hart, H. Ren, T. Wagner, P. Leubner, M. Muhlbauer, C. Brune, H. Buhmann, L. W. Molenkamp, and A. Yacoby, Nat. Phys. 10, 638 (2014).

[5] V. S. Pribiag, A. J. A. Beukman, F. Qu, M. C. Cassidy, C. Charpentier, W. Wegscheider, and L. P. Kouwenhoven, Nat. Nanotechnol. 10, 593 (2015).

[6] B. Baxevanis, V. P. Ostroukh, and C. W. J. Beenakker, Phys. Rev. B 91, 041409(R) (2015).

[7] F. K. de Vries, T. Timmerman, V. P. Ostroukh, J. van Veen, A. J. A. Beukman, F. Qu, M. Wimmer, B.-M. Nguyen, A. A. Kiselev, W. Yi, M. Sokolich, M. J. Manfra, C. M. Marcus, and L. P. Kouwenhoven, Phys. Rev. Lett. 120, 047702 (2018).

[8] A. Einstein, B. Podolsky, and N. Rosen, Phys. Rev. 47, 777 (1935).

[9] J. Klinovaja and D. Loss, Phys. Rev. B 90, 045118 (2014).

[10] E. Gaidamauskas, J. Paaske, and K. Flensberg, Phys. Rev. Lett. 112, 126402 (2014).

[11] P. Recher, E. V. Sukhorukov, and D. Loss, Phys. Rev. B 63, 165314 (2001).

[12] L. Hofstetter, S. Csonka, J. Nygard, and C. Schonenberger, Nature (London) 461, 960 (2009).

[13] P. Recher and D. Loss, Phys. Rev. B 65, 165327 (2002).

[14] S. Russo, M. Kroug, T. M. Klapwijk, and A. F. Morpurgo, Phys. Rev. Lett. 95, 027002 (2005).

[15] K. Sato, D. Loss, and Y. Tserkovnyak, Phys. Rev. B 85, 235433 (2012).

[16] M. de la Mata, R. Leturcq, S. R. Plissard, C. Rolland, C. Magen, J. Arbiol, and P. Caroff, Nano Lett. 16, 825 (2016).

[17] H. A. Nilsson, P. Caroff, C. Thelander, M. Larsson, J. B. Wagner, L.-E. Wernersson, L. Samuelson, and H. Q. Xu, Nano Lett. 9, 3151 (2009).

[18] D. Pan, D. X. Fan, N. Kang, J. H. Zhi, X. Z. Yu, H. Q. Xu, and J. H. Zhao, Nano Lett. 16, 834 (2016).

[19] J. Zhi, N. Kang, S. Li, D. Fan, F. Su, D. Pan, S. Zhao, J. Zhao, and H. Xu, Phys. Status Solidi B 256, 1800538 (2019).

[20] S. Gazibegovic, D. Car, H. Zhang, S. C. Balk, J. A. Logan, M. W. A. de Moor, M. C. Cassidy, R. Schmits, D. Xu, G. Wang, P. Krogstrup, R. L. M. O. het Veld, K. Zuo, Y. Vos, J. Shen, D. Bouman, B. Shojaei, D. Pennachio, J. S. Lee, P. J. van Veldhoven et al., Nature (London) 548, 434 (2017).

[21] E. Kreutz, E. Rickus, and N. Sotnik, Thin Solid Films 101, 153 (1983).

[22] A. Ritz and H. Lüth, J. Vac. Sci. Technol. B 3, 1153 (1985).
[23] L. J. Whitman, J. A. Stroscio, R. A. Dragoset, and R. J. Celotta, Phys. Rev. B 42, 7288 (1990).

[24] For devices I-III, the sulfur solution leaked underneath the etch mask, as apparent in the scanning electron microscope (SEM) images in Figs. 2(a), 2(b), and 5(b). Compared to devices IV-VII, that do not show this underetching [27], we did not observe a notable difference in the measurements.

[25] O. Gül, H. Zhang, F. K. de Vries, J. van Veen, K. Zuo, V. Mourik, S. Conesa-Boj, M. P. Nowak, D. J. van Woerkom, M. Quintero-Pérez, M. C. Cassidy, A. Geresdi, S. Koelling, D. Car, S. R. Plissard, E. P. A. M. Bakkers, and L. P. Kouwenhoven, Nano Lett. 17, 2690 (2017).

[26] See Supplemental Material at http://link.aps.org/supplemental/ 10.1103/PhysRevResearch.1.032031 for the theoretical models used, an overview of all measured devices, and additional measurements on other devices.

[27] V. Barzykin and A. M. Zagoskin, Superlatt. Microstruct. 25, 797 (1999).

[28] J. P. Heida, B. J. van Wees, T. M. Klapwijk, and G. Borghs, Phys. Rev. B 57, R5618 (1998).

[29] R. C. Dynes and T. A. Fulton, Phys. Rev. B 3, 3015 (1971).

[30] H.-Y. Hui, A. M. Lobos, J. D. Sau, and S. Das Sarma, Phys. Rev. B 90, 224517 (2014).

[31] T. Yokoyama, M. Eto, and Y. V. Nazarov, Phys. Rev. B 89, 195407 (2014).

[32] H. Meier, V. I. Fal'ko, and L. I. Glazman, Phys. Rev. B 93 , 184506 (2016).

[33] J. A. M. van Ostaay, A. R. Akhmerov, and C. W. J. Beenakker, Phys. Rev. B 83, 195441 (2011).

[34] R. Jacquet, J. Rech, T. Jonckheere, A. Zazunov, and T. Martin, Phys. Rev. B 92, 235429 (2015).

[35] J. Liu, H. Liu, J. Song, Q.-F. Sun, and X. C. Xie, Phys. Rev. B 96, 045401 (2017).

[36] Y. Sato, S. Matsuo, C.-H. Hsu, P. Stano, K. Ueda, Y. Takeshige, H. Kamata, J. S. Lee, B. Shojaei, K. Wickramasinghe, J. Shabani, C. Palmstrøm, Y. Tokura, D. Loss, and S. Tarucha, Phys. Rev. B 99, 155304 (2019).

[37] F. Chiodi, M. Ferrier, S. Guéron, J. C. Cuevas, G. Montambaux, F. Fortuna, A. Kasumov, and H. Bouchiat, Phys. Rev. B 86, 064510 (2012).

[38] M. Amado, A. Fornieri, F. Carillo, G. Biasiol, L. Sorba, V. Pellegrini, and F. Giazotto, Phys. Rev. B 87, 134506 (2013).

[39] F. K. de Vries, M. L. Sol, S. Gazibegovic, R. L. M. op het Veld, S. C. Balk, D. Car, E. P. A. M. Bakkers, L. P. Kouwenhoven, and J. Shen, Crossed Andreev Reflection in InSb Flake Josephson Junctions. 4TU.Centre for Research Data Dataset. https://doi.org/10.4121/uuid:8bcbeb5b9b0a-47d2-9264-5521aa748b64 (2019). 